\title{
The Cosmic Microwave Background Radiation
}

\author{
Eric Gawiser \\ Department of Physics, University of California, Berkeley, CA 94720 \\ and \\ Joseph Silk \\ Department of Physics, Astrophysics, 1 Keble Road, University of Oxford, \\ OX1 3NP, UK \\ and \\ Departments of Physics and Astronomy and Center for Particle \\ Astrophysics, University of California, Berkeley, CA 94720
}

\begin{abstract}
We summarize the theoretical and observational status of the study of the Cosmic Microwave Background radiation. Its thermodynamic spectrum is a robust prediction of the Hot Big Bang cosmology and has been confirmed observationally. There are now 76 observations of Cosmic Microwave Background anisotropy, which we present in a table with references. We discuss the theoretical origins of these anisotropies and explain the standard jargon associated with their observation.
\end{abstract}

\section{Origin of the Cosmic Background Radiation}

Our present understanding of the beginning of the universe is based upon the remarkably successful theory of the Hot Big Bang. We believe that our universe began about 15 billion years ago as a hot, dense, nearly uniform sea of radiation a minute fraction of its present size (formally an infinitesimal singularity). If inflation occurred in the first fraction of a second, the universe became matter dominated while expanding exponentially and then returned to radiation domination by the reheating caused by the decay of the inflaton. Baryonic matter formed within the first second, and the nucleosynthesis of the lightest elements took only a few minutes as the universe expanded and cooled. The baryons were in the form of plasma until about 300,000 years after the Big Bang, when the universe had cooled to a temperature near $3000 \mathrm{~K}$, sufficiently cool for protons to capture free electrons and form atomic hydrogen; this process is referred to as recombination. The recombination epoch occurred at

1 current address: Center for Astrophysics and Space Sciences, University of California at San Diego, La Jolla, CA 92037 
a redshift of 1100, meaning that the universe has grown over a thousand times larger since then. The ionization energy of a hydrogen atom is $13.6 \mathrm{eV}$, but recombination did not occur until the universe had cooled to a characteristic temperature $(\mathrm{kT})$ of $0.3 \mathrm{eV}$ (Padmanabhan, 1993). This delay had several causes. The high entropy of the universe made the rate of electron capture only marginally faster than the rate of photodissociation. Moreover, each electron captured directly into the ground state emits a photon capable of ionizing another newly formed atom, so it was through recombination into excited states and the cooling of the universe to temperatures below the ionization energy of hydrogen that neutral matter finally condensed out of the plasma. Until recombination, the universe was opaque to electromagnetic radiation due to scattering of the photons by free electrons. As recombination occurred, the density of free electrons diminished greatly, leading to the decoupling of matter and radiation as the universe became transparent to light.

The Cosmic Background Radiation (CBR) released during this era of decoupling has a mean free path long enough to travel almost unperturbed until the present day, where we observe it peaked in the microwave region of the spectrum as the Cosmic Microwave Background (CMB). We see this radiation today coming from the surface of last scattering (which is really a spherical shell of finite thickness) at a distance of nearly 15 billion light years. This Cosmic Background Radiation was predicted by the Hot Big Bang theory and discovered at an antenna temperature of 3K in 1964 by Penzias \& Wilson (1965). The number density of photons in the universe at a redshift $z$ is given by (Peebles, 1993)

$$
n_{\gamma}=420(1+z)^{3} \mathrm{~cm}^{-3}
$$

where $(1+z)$ is the factor by which the linear scale of the universe has expanded since then. The radiation temperature of the universe is given by $T=T_{0}(1+z)$ so it is easy to see how the conditions in the early universe at high redshifts were hot and dense.

The CBR is our best probe into the conditions of the early universe. Theories of the formation of large-scale structure predict the existence of slight inhomogeneities in the distribution of matter in the early universe which underwent gravitational collapse to form galaxies, galaxy clusters, and superclusters. These density inhomogeneities lead to temperature anisotropies in the CBR due to a combination of intrinsic temperature fluctuations and gravitational blue/redshifting of the photons leaving under/overdense regions. The DMR (Differential Microwave Radiometer) instrument of the Cosmic Background Explorer (COBE) satellite discovered primordial temperature fluctuations on angular scales larger than $7^{\circ}$ of order $\Delta T / T=10^{-5}$ (Smoot et al., 1992). Subsequent observations of the CMB have revealed temperature anisotropies on smaller angular scales which correspond to the physical scale of observed 
structures such as galaxies and clusters of galaxies.

\subsection{Thermalization}

There were three main processes by which this radiation interacted with matter in the first few hundred thousand years: Compton scattering, double Compton scattering, and thermal bremsstrahlung. The simplest interaction of matter and radiation is Compton scattering of a single photon off a free electron, $\gamma+e^{-} \rightarrow \gamma+e^{-}$. The photon will transfer momentum and energy to the electron if it has significant energy in the electron's rest frame. However, the scattering will be well approximated by Thomson scattering if the photon's energy in the rest frame of the electron is significantly less than the rest mass, $h \nu \ll m_{e} c^{2}$. When the electron is relativistic, the photon is blueshifted by roughly a factor $\gamma$ in energy when viewed from the electron rest frame, is then emitted at almost the same energy in the electron rest frame, and is blueshifted by another factor of $\gamma$ when retransformed to the observer's frame. Thus, energetic electrons can efficiently transfer energy to the photon background of the universe. This process is referred to as Inverse Compton scattering. The combination of cases where the photon gives energy to the electron and vice versa allows Compton scattering to generate thermal equilibrium (which is impossible in the Thomson limit of elastic scattering). Compton scattering conserves the number of photons. There exists a similar process, double Compton scattering, which produces (or absorbs) photons, $e^{-}+\gamma \leftrightarrow e^{-}+\gamma+\gamma$.

Another electromagnetic interaction which occurs in the plasma of the early universe is Coulomb scattering. Coulomb scattering establishes and maintains thermal equilibrium among the baryons of the photon-baryon fluid without affecting the photons. However, when electrons encounter ions they experience an acceleration and therefore emit electromagnetic radiation. This is called thermal bremsstrahlung or free-free emission. For an ion $X$, we have $e^{-}+X \leftrightarrow e^{-}+X+\gamma$. The interaction can occur in reverse because of the ability of the charged particles to absorb incoming photons; this is called freefree absorption. Each charged particle emits radiation, but the acceleration is proportional to the mass, so we can usually view the electron as being accelerated in the fixed Coulomb field of the much heavier ion. Bremsstrahlung is dominated by electric-dipole radiation (Shu, 1991) and can also produce and absorb photons.

The net effect is that Compton scattering is dominant for temperatures above $90 \mathrm{eV}$ whereas bremsstrahlung is the primary process between $90 \mathrm{eV}$ and 1 $\mathrm{eV}$. At temperatures above $1 \mathrm{keV}$, double Compton is more efficient than bremsstrahlung. All three processes occur faster than the expansion of the universe and therefore have an impact until decoupling. A static solution for 
Compton scattering is the Bose-Einstein distribution,

$$
f_{B E}=\frac{1}{e^{x+\mu}-1}
$$

where $\mu$ is a dimensionless chemical potential ( $\mathrm{Hu}, 1995)$. At high optical depths, Compton scattering can exchange enough energy to bring the photons to this Bose-Einstein equilibrium distribution. A Planckian spectrum corresponds to zero chemical potential, which will occur only when the number of photons and total energy are in the same proportion as they would be for a blackbody. Thus, unless the photon number starts out exactly right in comparison to the total energy in radiation in the universe, Compton scattering will only produce a Bose-Einstein distribution and not a blackbody spectrum. It is important to note, however, that Compton scattering will preserve a Planck distribution,

$$
f_{P}=\frac{1}{e^{x}-1}
$$

All three interactions will preserve a thermal spectrum if one is achieved at any point. It has long been known that the expansion of the universe serves to decrease the temperature of a blackbody spectrum,

$$
B_{\nu}=\frac{2 h \nu^{3} / c^{2}}{e^{h \nu / k T}-1}
$$

but keeps it thermal (Tolman, 1934). This occurs because both the frequency and temperature decrease as $(1+z)$ leaving $h \nu / k T$ unchanged during expansion. Although Compton scattering alone cannot produce a Planck distribution, such a distribution will remain unaffected by electromagnetic interactions or the universal expansion once it is achieved. A non-zero chemical potential will be reduced to zero by double Compton scattering and, later, bremsstrahlung which will create and absorb photons until the number density matches the energy and a thermal distribution of zero chemical potential is achieved. This results in the thermalization of the CBR at redshifts much greater than that of recombination.

Thermalization, of course, should only be able to create an equilibrium temperature over regions that are in causal contact. The causal horizon at the time of last scattering was relatively small, corresponding to a scale today of about $200 \mathrm{Mpc}$, or a region of angular extent of one degree on the sky. However, observations of the CMB show that it has an isotropic temperature on the sky to the level of one part in one hundred thousand! This is the origin of the Horizon Problem, which is that there is no physical mechanism expected 
in the early universe which can produce thermodynamic equilibrium on superhorizon scales. The inflationary universe paradigm (Guth, 1981; Linde, 1982; Albrecht \& Steinhardt, 1982) solves the Horizon Problem by postulating that the universe underwent a brief phase of exponential expansion during the first second after the Big Bang, during which our entire visible Universe expanded out of a region small enough to have already achieved thermal equilibrium.

\section{CMB Spectrum}

The CBR is the most perfect blackbody ever seen, according to the FIRAS (Far InfraRed Absolute Spectrometer) instrument of COBE, which measured a temperature of $T_{0}=2.726 \pm 0.010 \mathrm{~K}$ (Mather et al., 1994). The theoretical prediction that the CBR will have a blackbody spectrum appears to be confirmed by the FIRAS observation (see Figure 11). But this is not the end of the story. FIRAS only observed the peak of the blackbody. Other experiments have mapped out the Rayleigh-Jeans part of the spectrum at low frequency. Most are consistent with a $2.73 \mathrm{~K}$ blackbody, but some are not. It is in the low-frequency limit that the greatest spectral distortions might occur because a Bose-Einstein distribution differs from a Planck distribution there. However, double Compton and bremsstrahlung are most effective at low frequencies so strong deviations from a blackbody spectrum are not generally expected.

Spectral distortions in the Wien tail of the spectrum are quite difficult to detect due to the foreground signal from interstellar dust at those high frequencies. For example, broad emission lines from electron capture at recombination are predicted in the Wien tail but cannot be distinguished due to foreground contamination (White et al., 1994). However, because the energy generated by star formation and active galactic nuclei is absorbed by interstellar dust in all galaxies and then re-radiated in the far-infrared, we expect to see an isotropic Far-Infrared Background (FIRB) which dominates the CMB at frequencies above a few hundred GHz. This FIRB has now been detected in FIRAS data (Puget et al., 1996; Burigana \& Popa, 1998; Fixsen et al., 1998) and in data from the COBE DIRBE instrument (Schlegel et al., 1998; Dwek et al., 1998).

Although Compton, double Compton, and bremsstrahlung interactions occur frequently until decoupling, the complex interplay between them required to thermalize the CBR spectrum is ineffective at redshifts below $10^{7}$. This means that any process after that time which adds a significant portion of energy to the universe will lead to a spectral distortion today. Neutrino decays during this epoch should lead to a Bose-Einstein rather than a Planck distribution, and this allows the FIRAS observations to set constraints on the decay of neutrinos and other particles in the early universe (Kolb \& Turner, 1990). The apparent impossibility of thermalizing radiation at low redshift makes 


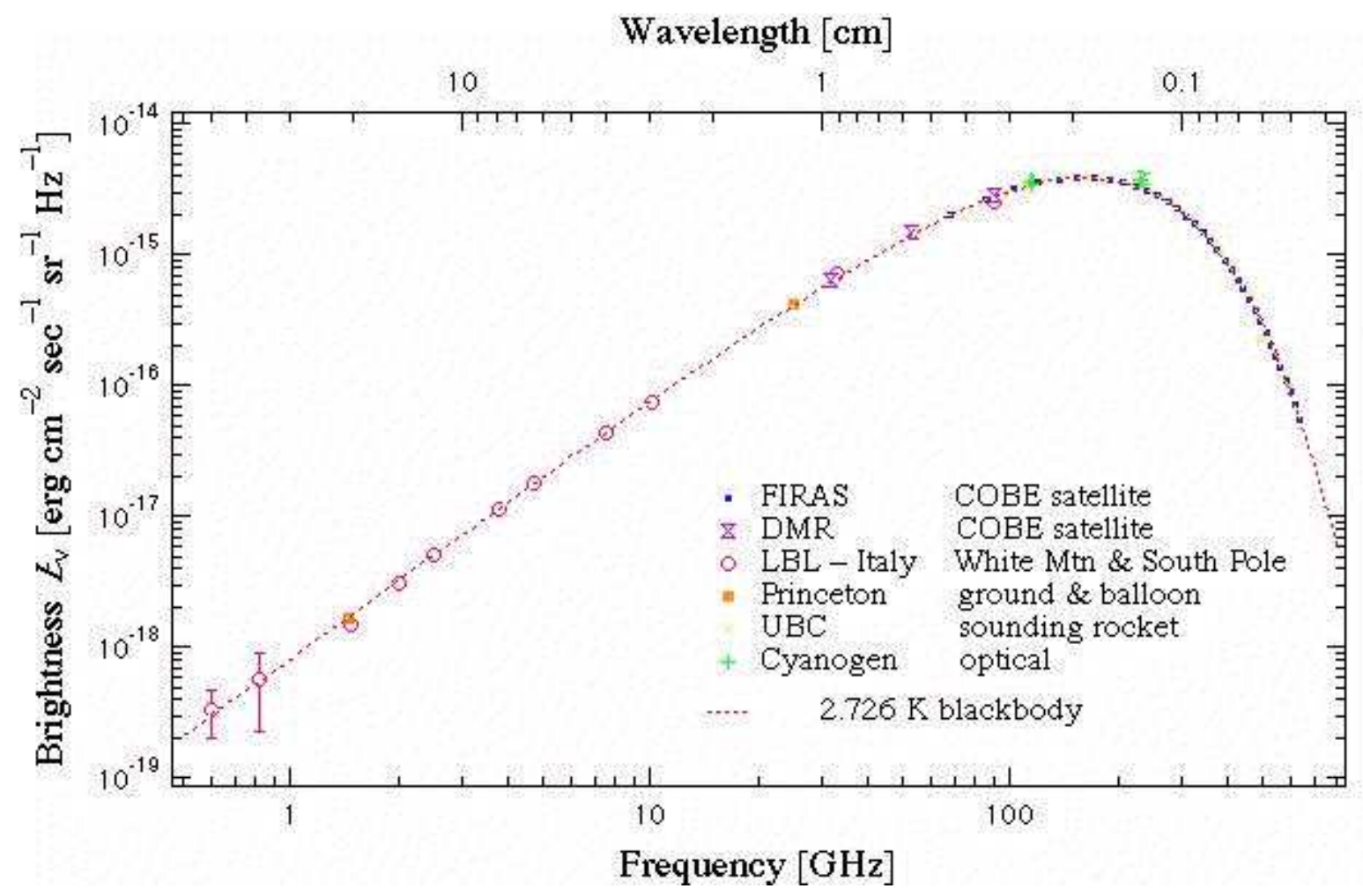

Fig. 1. Measurements of the CMB spectrum.

the blackbody nature of the CBR strong evidence that it did originate in the early universe and as a result serves to support the Big Bang theory.

The process of Compton scattering can cause spectral distortions if it is too late for double Compton and bremsstrahlung to be effective. In general, lowfrequency photons will be shifted to higher frequencies, thereby decreasing the number of photons in the Rayleigh-Jeans region and enhancing the Wien tail. This is referred to as a Compton- $y$ distortion and it is described by the parameter

$$
y=\int \frac{T_{e}(t)}{m_{e}} \sigma n_{e}(t) d t
$$

The apparent temperature drop in the long-wavelength limit is

$$
\frac{\delta T}{T}=-2 y
$$

The most important example of this is Compton scattering of photons off hot electrons in galaxy clusters, called the Sunyaev-Zel'dovich (SZ) effect. The electrons transfer energy to the photons, and the spectral distortion results from the sum of all of the scatterings off electrons in thermal motion, each of which has a Doppler shift. The SZ effect from clusters can yield a distortion 
of $y \simeq 10^{-5}-10^{-3}$ and these distortions have been observed in several rich clusters of galaxies. The FIRAS observations place a constraint on any fullsky Comptonization by limiting the average $y$-distortion to $y<2.5 \times 10^{-5}$ (Hu, 1995). The integrated $y$-distortion predicted from the SZ effect of galaxy clusters and large-scale structure is over a factor of ten lower than this observational constraint (Refregier et al., 1998) but that from "cocoons" of radio galaxies (Yamada et al., 1999) is predicted to be of the same order. A kinematic SZ effect is caused by the bulk velocity of the cluster; this is a small effect which is very difficult to detect for individual clusters but will likely be measured statistically by the Planck satellite.

\section{CMB Anisotropy}

The temperature anisotropy at a point on the sky $(\theta, \phi)$ can be expressed in the basis of spherical harmonics as

$$
\frac{\Delta T}{T}(\theta, \phi)=\sum_{\ell m} a_{\ell m} Y_{\ell m}(\theta, \phi) .
$$

A cosmological model predicts the variance of the $a_{\ell m}$ coefficients over an ensemble of universes (or an ensemble of observational points within one universe, if the universe is ergodic). The assumptions of rotational symmetry and Gaussianity allow us to express this ensemble average in terms of the multipoles $C_{\ell}$ as

$$
\left\langle a_{\ell m}^{*} a_{\ell^{\prime} m^{\prime}}\right\rangle \equiv C_{\ell} \delta_{\ell^{\prime} \ell} \delta_{m^{\prime} m}
$$

The predictions of a cosmological model can be expressed in terms of $C_{\ell}$ alone if that model predicts a Gaussian distribution of density perturbations, in which case the $a_{\ell m}$ will have mean zero and variance $C_{\ell}$.

The temperature anisotropies of the CMB detected by COBE are believed to result from inhomogeneities in the distribution of matter at the epoch of recombination. Because Compton scattering is an isotropic process in the electron rest frame, any primordial anisotropies (as opposed to inhomogeneities) should have been smoothed out before decoupling. This lends credence to the interpretation of the observed anisotropies as the result of density perturbations which seeded the formation of galaxies and clusters. The discovery of temperature anisotropies by COBE provides evidence that such density inhomogeneities existed in the early universe, perhaps caused by quantum fluctuations in the scalar field of inflation or by topological defects resulting from a phase transition (see Kamionkowski \& Kosowsky, 1999 for a detailed 
review of inflationary and defect model predictions for CMB anisotropies). Gravitational collapse of these primordial density inhomogeneities appears to have formed the large-scale structures of galaxies, clusters, and superclusters that we observe today.

On large (super-horizon) scales, the anisotropies seen in the CMB are produced by the Sachs-Wolfe effect (Sachs \& Wolfe, 1967).

$$
\left(\frac{\Delta T}{T}\right)_{S W}=\left.\mathbf{v} \cdot \mathbf{e}\right|_{o} ^{e}-\left.\Phi\right|_{o} ^{e}+\frac{1}{2} \int_{o}^{e} h_{\rho \sigma, 0} n^{\rho} n^{\sigma} d \xi
$$

where the first term is the net Doppler shift of the photon due to the relative motion of emitter and observer, which is referred to as the kinematic dipole. This dipole, first observed by Smoot et al. (1977), is much larger than other $\mathrm{CMB}$ anisotropies and is believed to reflect the motion of the Earth relative to the average reference frame of the CMB. Most of this motion is due to the peculiar velocity of the Local Group of galaxies. The second term represents the gravitational redshift due to a difference in gravitational potential between the site of photon emission and the observer. The third term is called the Integrated Sachs-Wolfe (ISW) effect and is caused by a non-zero time derivative of the metric along the photon's path of travel due to potential decay, gravitational waves, or non-linear structure evolution (the Rees-Sciama effect). In a matter-dominated universe with scalar density perturbations the integral vanishes on linear scales. This equation gives the redshift from emission to observation, but there is also an intrinsic $\Delta T / T$ on the last-scattering surface due to the local density of photons. For adiabatic perturbations, we have (White \& Hu, 1997) an intrinsic

$$
\frac{\Delta T}{T}=\frac{1}{3} \frac{\delta \rho}{\rho}=\frac{2}{3} \Phi .
$$

Putting the observer at $\Phi=0$ (the observer's gravitational potential merely adds a constant energy to all CMB photons) this leads to a net Sachs-Wolfe effect of $\Delta T / T=-\Phi / 3$ which means that overdensities lead to cold spots in the CMB.

\subsection{Small-angle anisotropy}

Anisotropy measurements on small angular scales $\left(0.1\right.$ to $\left.1^{\circ}\right)$ are expected to reveal the so-called first acoustic peak of the CMB power spectrum. This peak in the anisotropy power spectrum corresponds to the scale where acoustic oscillations of the photon-baryon fluid caused by primordial density inhomo- 


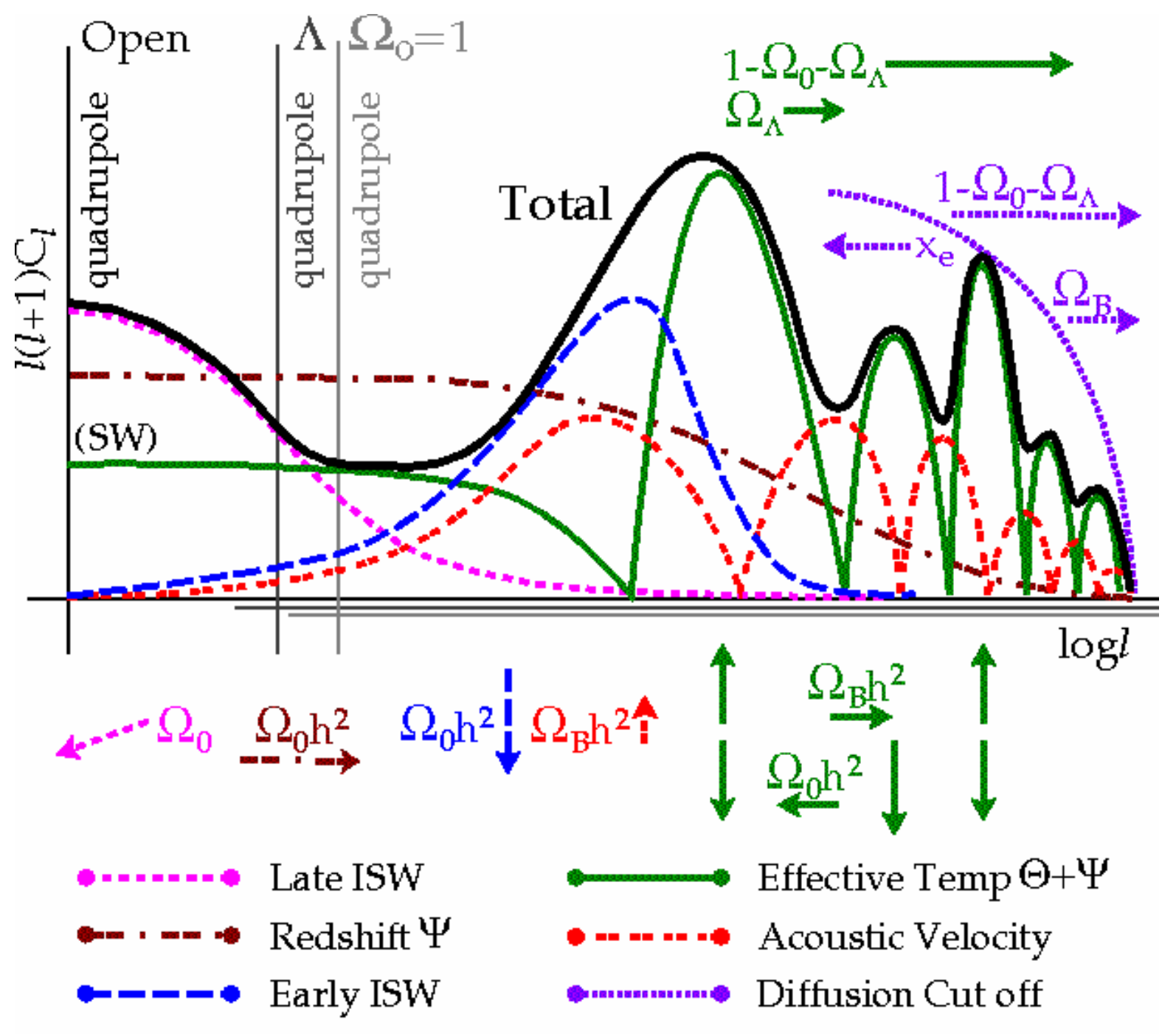

Hu, Sugiyama, \& Silk (1995)

Fig. 2. Dependence of CMB anisotropy power spectrum on cosmological parameters.

geneities are just reaching their maximum amplitude at the surface of last scattering i.e. the sound horizon at recombination. Further acoustic peaks occur at scales that are reaching their second, third, fourth, etc. antinodes of oscillation.

Figure 2 (from $\mathrm{Hu}$ et al., 1997) shows the dependence of the CMB anisotropy power spectrum on a number of cosmological parameters. The acoustic oscillations in density (light solid line) are sharp here because they are really being plotted against spatial scales, which are then smoothed as they are projected through the last-scattering surface onto angular scales. The troughs in the density oscillations are filled in by the 90-degree-out-of-phase velocity oscillations (this is a Doppler effect but does not correspond to the net peaks, which are best referred to as acoustic peaks rather than Doppler peaks). The origin 
of this plot is at a different place for different values of the matter density and the cosmological constant; the negative spatial curvature of an open universe makes a given spatial scale correspond to a smaller angular scale. The Integrated Sachs-Wolfe (ISW) effect occurs whenever gravitational potentials decay due to a lack of matter dominance. Hence the early ISW effect occurs just after recombination when the density of radiation is still considerable and serves to broaden the first acoustic peak at scales just larger than the horizon size at recombination. And for a present-day matter density less than critical, there is a late ISW effect that matters on very large angular scales it is greater in amplitude for open universes than for lambda-dominated because matter domination ends earlier in an open universe for the same value of the matter density today. The late ISW effect should correlate with largescale structures that are otherwise detectable at $z \sim 1$, and this allows the CMB to be cross-correlated with observations of the X-ray background to determine $\Omega$ (Crittenden \& Turok, 1996; Kamionkowski, 1996; Boughn et al. 1998; Kamionkowski \& Kinkhabwala, 1999) or with observations of large-scale structure to determine the bias of galaxies (Suginohara et al., 1998).

For a given model, the location of the first acoustic peak can yield information about $\Omega$, the ratio of the density of the universe to the critical density needed to stop its expansion. For adiabatic density perturbations, the first acoustic peak will occur at $\ell=220 \Omega^{-1 / 2}$ (Kamionkowski et al., 1994). The ratio of $\ell$ values of the peaks is a robust test of the nature of the density perturbations; for adiabatic perturbations these will have ratio 1:2:3:4 whereas for isocurvature perturbations the ratio should be 1:3:5:7 ( $\mathrm{Hu} \&$ White, 1996). A mixture of adiabatic and isocurvature perturbations is possible, and this test should reveal it.

As illustrated in Figure 2, the amplitude of the acoustic peaks depends on the baryon fraction $\Omega_{b}$, the matter density $\Omega_{0}$, and Hubble's constant $H_{0}=100 \mathrm{~h}$ $\mathrm{km} / \mathrm{s} / \mathrm{Mpc}$. A precise measurement of all three acoustic peaks can reveal the fraction of hot dark matter and even potentially the number of neutrino species (Dodelson et al., 1996). Figure 2 i shows the envelope of the CMB anisotropy damping tail on arcminute scales, where the fluctuations are decreased due to photon diffusion (Silk, 1967) as well as the finite thickness of the last-scattering surface. This damping tail is a sensitive probe of cosmological parameters and has the potential to break degeneracies between models which explain the larger-scale anisotropies ( $\mathrm{Hu} \&$ White, 1997b; Metcalf \& Silk, 1998). The characteristic angular scale for this damping is given by $1.8^{\prime} \Omega_{B}^{-1 / 2} \Omega_{0}^{3 / 4} h^{-1 / 2}$ (White et al., 1994).

There is now a plethora of theoretical models which predict the development of primordial density perturbations into microwave background anisotropies. These models differ in their explanation of the origin of density inhomogeneities (inflation or topological defects), the nature of the dark matter (hot, 
cold, baryonic, or a mixture of the three), the curvature of the universe $(\Omega)$, the value of the cosmological constant $(\Lambda)$, the value of Hubble's constant, and the possibility of reionization which wholly or partially erased temperature anisotropies in the CMB on scales smaller than the horizon size. Available data does not allow us to constrain all (or even most) of these parameters, so analyzing current CMB anisotropy data requires a model-independent approach. It seems reasonable to view the mapping of the acoustic peaks as a means of determining the nature of parameter space before going on to fitting cosmological parameters directly.

\subsection{Reionization}

The possibility that post-decoupling interactions between ionized matter and the CBR have affected the anisotropies on scales smaller than those measured by COBE is of great significance for current experiments. Reionization is inevitable but its effect on anisotropies depends significantly on when it occurs (see Haimann \& Knox, 1999 for a review). Early reionization leads to a larger optical depth and therefore a greater damping of the anisotropy power spectrum due to the secondary scattering of CMB photons off of the newly free electrons. For a universe with critical matter density and constant ionization fraction $x_{e}$, the optical depth as a function of redshift is given by (White et al. 1994)

$$
\tau \simeq 0.035 \Omega_{B} h x_{e} z^{3 / 2}
$$

which allows us to determine the redshift of reionization $z_{*}$ at which $\tau=1$,

$$
z_{*} \simeq 69\left(\frac{h}{0.5}\right)^{-\frac{2}{3}}\left(\frac{\Omega_{B}}{0.1}\right)^{-\frac{2}{3}} x_{e}^{-\frac{2}{3}} \Omega^{\frac{1}{3}}
$$

where the scaling with $\Omega$ applies to an open universe only. At scales smaller than the horizon size at reionization, $\Delta T / T$ is reduced by the factor $e^{-\tau}$.

Attempts to measure the temperature anisotropy on angular scales of less than a degree which correspond to the size of galaxies could have led to a surprise; if the universe was reionized after recombination to the extent that the CBR was significantly scattered at redshifts less than 1100, the small-scale primordial anisotropies would have been washed out. To have an appreciable optical depth for photon-matter interaction, reionization cannot have occurred much later than a redshift of 20 (Padmanabhan, 1993). Large-scale anisotropies such as those seen by COBE are not expected to be affected by reionization because they encompass regions of the universe which were not yet in causal 
contact even at the proposed time of reionization. However, the apparently high amplitiude of degree-scale anisotropies is a strong argument against the possibility of early $(z \geq 50)$ reionization. On arc-minute scales, the interaction of photons with reionized matter is expected to have eliminated the primordial anisotropies and replaced them with smaller secondary anisotropies from this new surface of last scattering (the Ostriker-Vishniac effect and patchy reionization, see next section).

\subsection{Secondary Anisotropies}

Secondary CMB anisotropies occur when the photons of the Cosmic Microwave Background radiation are scattered after the original last-scattering surface (see Refregier, 1999 for a review). The shape of the blackbody spectrum can be altered through inverse Compton scattering by the thermal SunyaevZel'dovich (SZ) effect (Sunyaev \& Zeldovich, 1972). The effective temperature of the blackbody can be shifted locally by a doppler shift from the peculiar velocity of the scattering medium (the kinetic SZ and Ostriker-Vishniac effects, Ostriker \& Vishniac, 1986) as well as by passage through the changing gravitational potential caused by the collapse of nonlinear structure (the ReesSciama effect, Rees \& Sciama, 1968) or the onset of curvature or cosmological constant domination (the Integrated Sachs-Wolfe effect). Several simulations of the impact of patchy reionization have been performed (Aghanim et al. 1996; [Knox et al., 1998; Gruzinov \& Hu, 1998; Peebles \& Juszkiewicz, 1998). The SZ effect itself is independent of redshift, so it can yield information on clusters at much higher redshift than does X-ray emission. However, nearly all clusters are unresolved for $10^{\prime}$ resolution so higher-redshift clusters occupy less of the beam and therefore their SZ effect is in fact dimmer. In the $4.5^{\prime}$ channels of Planck this will no longer be true, and the SZ effect can probe cluster abundance at high redshift. An additional secondary anisotropy is that caused by gravitational lensing (see e.g. Cayon et al., 1993, 1994; Metcalf \& Silk, 1997; Martinez-Gonzalez et al., 1997). Gravitational lensing imprints slight non-Gaussianity in the CMB from which it might be possible to deter-

mine the matter power spectrum (Seljak \& Zaldarriaga, 1998; Zaldarriaga \& Seljak, 1998).

\subsection{Polarization Anisotropies}

Polarization of the Cosmic Microwave Background radiation (Kosowsky, 1994; Kamionkowski et al., 1997; Zaldarriaga \& Seljak, 1997) arises due to local quadrupole anisotropies at each point on the surface of last scattering (see $\mathrm{Hu}$ \& White, 1997a for a review). Scalar (density) perturbations generate curl- 
free (electric mode) polarization only, but tensor (gravitational wave) perturbations can generate divergence-free (magnetic mode) polarization. Hence the polarization of the CMB is a potentially useful probe of the level of gravitational waves in the early universe (Seljak \& Zaldarriaga, 1997; Kamionkowski \& Kosowsky, 1998), especially since current indications are that the large-scale primary anisotropies seen by COBE do not contain a measurable fraction of tensor contributions (Gawiser \& Silk, 1998). A thorough review of gravity waves and CMB polarization is given by Kamionkowski \& Kosowsky (1999).

\subsection{Gaussianity of the $C M B$ anisotropies}

The processes turning density inhomogeneities into CMB anisotropies are linear, so cosmological models that predict gaussian primordial density inhomogeneities also predict a gaussian distribution of CMB temperature fluctuations. Several techniques have been developed to test COBE and future datasets for deviations from gaussianity (e.g. Kogut et al., 1996b; Ferreira \& Magueijo, 1997; Ferreira et al., 1997). Most tests have proven negative, but a few claims of non-gaussianity have been made. Gaztañaga et al. (1998) found a very marginal indication of non-gaussianity in the spread of results for degree-scale CMB anisotropy observations being greater than the expected sample variances. Ferreira et al. (1998) have claimed a detection of non-gaussianity at multipole $\ell=16$ using a bispectrum statistic, and Pando et al. (1998) find a non-gaussian wavelet coefficient correlation on roughly $15^{\circ}$ scales in the North Galactic hemisphere. Both of these methods produce results consistent with gaussianity, however, if a particular area of several pixels is eliminated from the dataset (Bromley \& Tegmark, 1999). A true sky signal should be larger than several pixels so instrument noise is the most likely source of the nongaussianity. A different area appears to cause each detection, giving evidence that the COBE dataset had non-gaussian instrument noise in at least two areas of the sky.

\subsection{Foreground contamination}

Of particular concern in measuring CMB anisotropies is the issue of foreground contamination. Foregrounds which can affect CMB observations include galactic radio emission (synchrotron and free-free), galactic infrared emission (dust), extragalactic radio sources (primarily elliptical galaxies, active galactic nuclei, and quasars), extragalactic infrared sources (mostly dusty spirals and high-redshift starburst galaxies), and the Sunyaev-Zel'dovich effect from hot gas in galaxy clusters. The COBE team has gone to great lengths to analyze their data for possible foreground contamination and routinely elimi- 
nates everything within about $30^{\circ}$ of the galactic plane.

An instrument with large resolution such as COBE is most sensitive to the diffuse foreground emission of our Galaxy, but small-scale anisotropy experiments need to worry about extragalactic sources as well. Because foreground and CMB anisotropies are assumed to be uncorrelated, they should add in quadrature, leading to an increase in the measurement of CMB anisotropy power. Most CMB instruments, however, can identify foregrounds by their spectral signature across multiple frequencies or their display of the beam response characteristic of a point source. This leads to an attempt at foreground subtraction, which can cause an underestimate of CMB anisotropy if some true signal is subtracted along with the foreground. Because they are now becoming critical, extragalactic foregrounds have been studied in detail (Toffolatt] et al., 1998; Refregier et al., 1998; Gawiser \& Smoot, 1997; Sokasian et al. 1998; Gawiser et al., 1998). The Wavelength-Oriented Microwave Background Analysis Team (WOMBAT, see Gawiser et al., 1998; Jaffe et al., 1999) has made Galactic and extragalactic foreground predictions and full-sky simulations of realistic CMB skymaps containing foreground contamination available to the public (see http://astro.berkeley.edu/wombat). One of these CMB simulations is shown in Figure 3. Tegmark et al. (1999) used a Fisher matrix analysis to show that simultaneously estimating foreground model parameters and cosmological parameters can lead to a factor of a few degradation in the precision with which the cosmological parameters can be determined by CMB anisotropy observations, so foreground prediction and subtraction is likely to be an important aspect of future CMB data analysis.

Foreground contamination may turn out to be a serious problem for measurements of CMB polarization anisotropy. While free-free emission is unpolarized, synchrotron radiation displays a linear polarization determined by the coherence of the magnetic field along the line of sight; this is typically on the order of $10 \%$ for Galactic synchrotron and between 5 and $10 \%$ for flat-spectrum radio sources. The CMB is expected to show a large-angular scale linear polarization of about $10 \%$, so the prospects for detecting polarization anisotropy are no worse than for temperature anisotropy although higher sensitivity is required. However, the small-angular scale electric mode of linear polarization which is a probe of several cosmological parameters and the magnetic mode that serves as a probe of tensor perturbations are expected to have much lower amplitude and may be swamped by foreground polarization. Thermal and spinning dust grain emission can also be polarized. It may turn out that dust emission is the only significant source of circularly polarized microwave photons since the CMB cannot have circular polarization. 


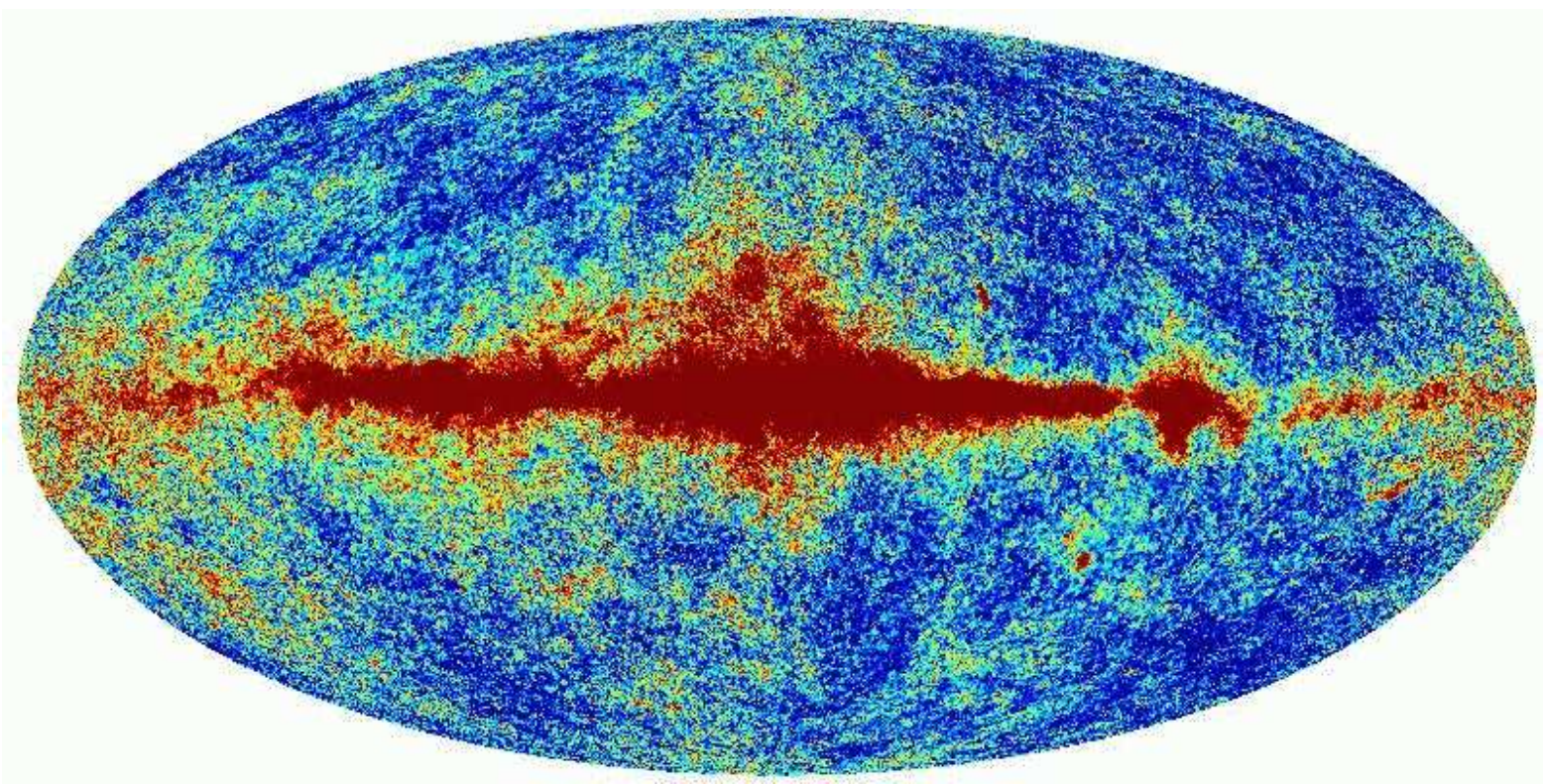

Fig. 3. WOMBAT Challenge simulation of CMB anisotropy map that might be observed by the MAP satellite at $90 \mathrm{GHz}, 13$ ' resolution, containing $\mathrm{CMB}$, instrument noise, and foreground contamination. The resolution is degraded by the pixelization of your monitor or printer.

\section{Cosmic Microwave Background Anisotropy Observations}

Since the COBE DMR detection of CMB anisotropy (Smoot et al., 1992), there have been over thirty additional measurements of anisotropy on angular scales ranging from $7^{\circ}$ to 0.3 , and upper limits have been set on smaller scales.

The COBE DMR observations were pixelized into a skymap, from which it is possible to analyze any particular multipole within the resolution of the DMR. Current small angular scale CMB anisotropy observations are insensitive to both high $\ell$ and low $\ell$ multipoles because they cannot measure features smaller than their resolution and are insensitive to features larger than the size of the patch of sky observed. The next satellite mission, NASA's Microwave Anisotropy Probe (MAP), is scheduled for launch in Fall 2000 and will map angular scales down to 0.2 with high precision over most of the sky. An even more precise satellite, ESA's Planck, is scheduled for launch in 2007. Because COBE observed such large angles, the DMR data can only constrain the amplitude $A$ and index $n$ of the primordial power spectrum in wave number $k$, $P_{p}(k)=A k^{n}$, and these constraints are not tight enough to rule out very many classes of cosmological models.

Until the next satellite is flown, the promise of microwave background anisotropy measurements to measure cosmological parameters rests with a series of groundbased and balloon-borne anisotropy instruments which have already pub- 
lished results (shown in Figure 1 ) or will report results in the next few years (MAXIMA, BOOMERANG, TOPHAT, ACE, MAT, VSA, CBI, DASI, see Lee et al., 1999 and Halpern \& Scott, 1999). Because they are not satellites, these instruments face the problems of shorter observing times and less sky coverage, although significant progress has been made in those areas. They fall into three categories: high-altitude balloons, interferometers, and other ground-based instruments. Past, present, and future balloon-borne instruments are FIRS, MAX, MSAM, ARGO, BAM, MAXIMA, QMAP, HACME, BOOMERANG, TOPHAT, and ACE. Ground-based interferometers include CAT, JBIAC, SUZIE, BIMA, ATCA, VLA, VSA, CBI, and DASI, and other ground-based instruments are TENERIFE, SP, PYTHON, SK, OVRO/RING, VIPER, MAT/TOCO, IACB, and WD. Taken as a whole, they have the potential to yield very useful measurements of the radiation power spectrum of the CMB on degree and subdegree scales. Ground-based non-interferometers have to discard a large fraction of data and undergo careful further data reduction to eliminate atmospheric contamination. Balloon-based instruments need to keep a careful record of their pointing to reconstruct it during data analysis. Interferometers may be the most promising technique at present but they are the least developed, and most instruments are at radio frequencies and have very narrow frequency coverage, making foreground contamination a major concern. In order to use small-scale CMB anisotropy measurements to constrain cosmological models we need to be confident of their validity and to trust the error bars. This will allow us to discard badly contaminated data and to give greater weight to the more precise measurements in fitting models. Correlated noise is a great concern for instruments which lack a rapid chopping because the $1 / f$ noise causes correlations on scales larger than the beam in a way that can easily mimic CMB anisotropies. Additional issues are sample variance caused by the combination of cosmic variance and limited sky coverage and foreground contamination.

Figure 1 shows our compilation of CMB anisotropy observations without adding any theoretical curves to bias the eye ${ }^{2}$. It is clear that a straight line is a poor but not implausible fit to the data. There is a clear rise around $\ell=100$ and then a drop by $\ell=1000$. This is not yet good enough to give a clear determination of the curvature of the universe, let alone fit several cosmological parameters. However, the current data prefer adiabatic structure formation models over isocurvature models (Gawiser \& Silk, 1998). If analysis is restricted to adiabatic CDM models, a value of the total density near critical is preferred (Dodelson \& Knox, 1999).

$\overline{2}$ This figure and our compilation of CMB anisotropy observations are available at http://mamacass.ucsd.edu/people/gawiser/cmb.htm; CMB observations have also been compiled by Smoot \& Scott (1998) and at http://www.hep.upenn.edu/ ${ }^{\sim} \max / \mathrm{cmb} /$ experiments.htm] and http://www.cita.utoronto.ca/ $\mathrm{knox} /$ radical.htm] 


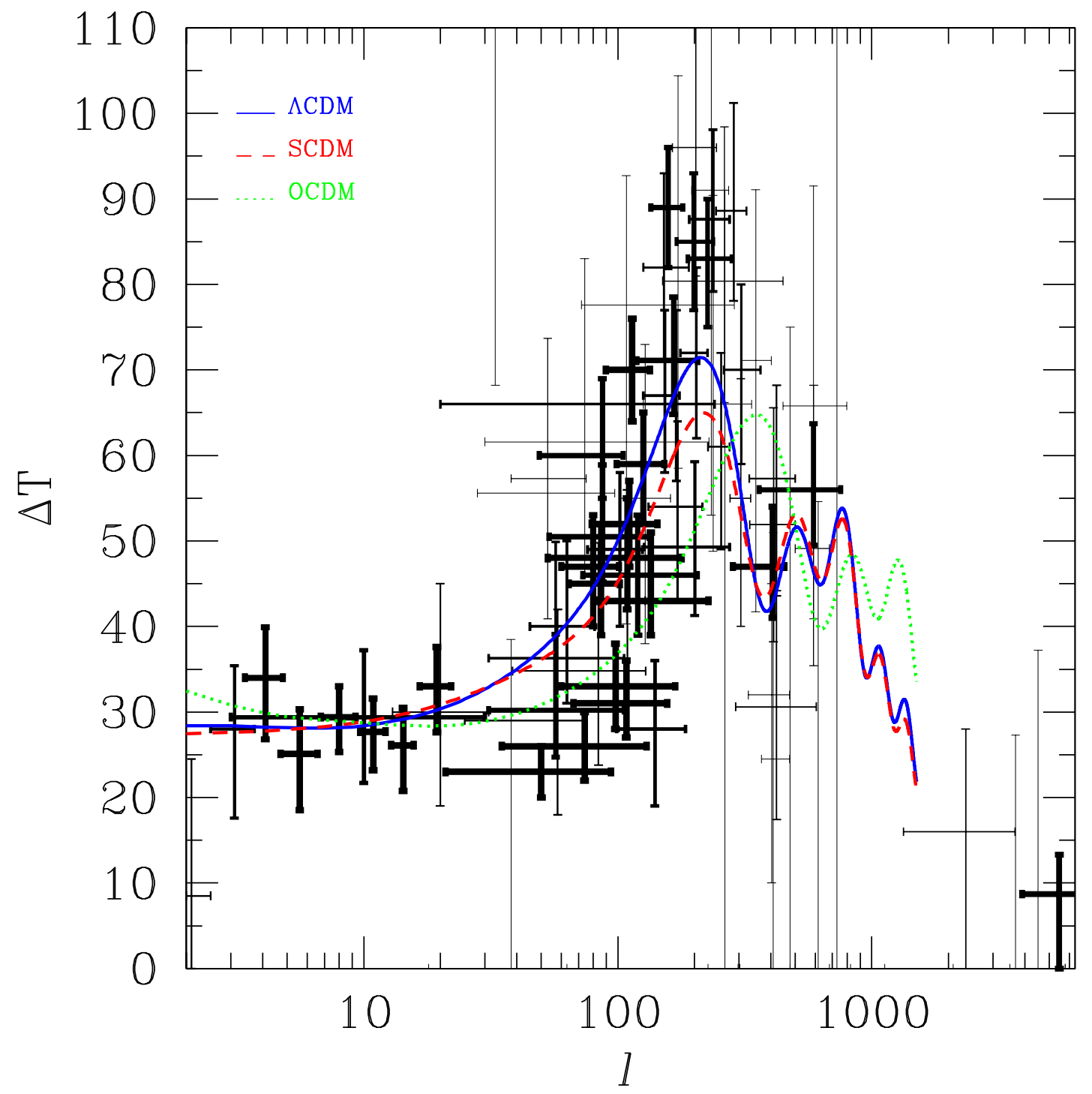

Fig. 4. Compilation of CMB Anisotropy observations. Vertical error bars represent $1 \sigma$ uncertainties and horizontal error bars show the range from $\ell_{\min }$ to $\ell_{\max }$ of Table 1. The line thickness is inversely proportional to the variance of each measurement, emphasizing the tighter constraints. All three models are consistent with the upper limits at the far right, but the Open CDM model (dotted) is a poor fit to the data, which prefer models with an acoustic peak near $\ell=200$ with an amplitude close to that of $\Lambda$ CDM (solid).

\subsection{Window Functions}

The sensitivity of these instruments to various multipoles is called their window function. These window functions are important in analyzing anisotropy measurements because the small-scale experiments do not measure enough of the sky to produce skymaps like COBE. Rather they yield a few "band-power" 
measurements of rms temperature anisotropy which reflect a convolution over the range of multipoles contained in the window function of each band. Some instruments can produce limited skymaps (White \& Bunn, 1995). The window function $W_{\ell}$ shows how the total power observed is sensitive to the anisotropy on the sky as a function of angular scale:

$$
\text { Power }=\frac{1}{4 \pi} \sum_{\ell}(2 \ell+1) C_{\ell} W_{\ell}=\frac{1}{2}\left(\Delta T / T_{C M B}\right)^{2} \sum_{\ell} \frac{2 \ell+1}{\ell(\ell+1)} W_{\ell}
$$

where the COBE normalization is $\Delta T=27.9 \mu \mathrm{K}$ and $T_{C M B}=2.73 \mathrm{~K}$ (Bennett et al., 1996). This allows the observations of broad-band power to be reported as observations of $\Delta T$, and knowing the window function of an instrument one can turn the predicted $C_{\ell}$ spectrum of a model into the corresponding prediction for $\Delta T$. This "band-power" measurement is based on the standard definition that for a "flat" power spectrum, $\Delta T=\left(\ell(\ell+1) C_{\ell}\right)^{1 / 2} T_{C M B} /(2 \pi)$ (flat actually means that $\ell(\ell+1) C_{\ell}$ is constant).

The autocorrelation function for measured temperature anisotropies is a convolution of the true expectation values for the anisotropies and the window function. Thus we have (White \& Srednicki, 1995)

$$
\left\langle\frac{\Delta T}{T}\left(\hat{n}_{1}\right) \frac{\Delta T}{T}\left(\hat{n}_{2}\right)\right\rangle=\frac{1}{4 \pi} \sum_{\ell=1}^{\infty}(2 \ell+1) C_{\ell} W_{\ell}\left(\hat{n}_{1}, \hat{n}_{2}\right),
$$

where the symmetric beam shape that is typically assumed makes $W_{\ell}$ a function of separation angle only. In general, the window function results from a combination of the directional response of the antenna, the beam position as a function of time, and the weighting of each part of the beam trajectory in producing a temperature measurement (White \& Srednicki, 1995). Strictly speaking, $W_{\ell}$ is the diagonal part of a filter function $W_{\ell \ell^{\prime}}$ that reflects the coupling of various multipoles due to the non-orthogonality of the spherical harmonics on a cut sky and the observing strategy of the instrument (Knox. 1999). It is standard to assume a Gaussian beam response of width $\sigma$, leading to a window function

$$
W_{\ell}=\exp \left[-\ell(\ell+1) \sigma^{2}\right]
$$

The low- $\ell$ cutoff introduced by a 2 -beam differencing setup comes from the window function (White et al., 1994)

$$
W_{\ell}=2\left[1-P_{\ell}(\cos \theta)\right] \exp \left[-\ell(\ell+1) \sigma^{2}\right] .
$$




\subsection{Sample and Cosmic Variance}

The multipoles $C_{\ell}$ can be related to the expected value of the spherical harmonic coefficients by

$$
\left\langle\sum_{m} a_{\ell m}^{2}\right\rangle=(2 \ell+1) C_{\ell}
$$

since there are $(2 \ell+1) a_{\ell m}$ for each $\ell$ and each has an expected autocorrelation of $C_{\ell}$. In a theory such as inflation, the temperature fluctuations follow a Gaussian distribution about these expected ensemble averages. This makes the $a_{\ell m}$ Gaussian random variables, resulting in a $\chi_{2 \ell+1}^{2}$ distribution for $\sum_{m} a_{\ell m}^{2}$. The width of this distribution leads to a cosmic variance in the estimated $C_{\ell}$

of $\sigma_{c v}^{2}=\left(\ell+\frac{1}{2}\right)^{-\frac{1}{2}} C_{\ell}$, which is much greater for small $\ell$ than for large $\ell$ (unless $C_{\ell}$ is rising in a manner highly inconsistent with theoretical expectations). So, although cosmic variance is an unavoidable source of error for anisotropy measurements, it is much less of a problem for small scales than for COBE.

Despite our conclusion that cosmic variance is a greater concern on large angular scales, Figure 4 shows a tremendous variation in the level of anisotropy measured by small-scale experiments. Is this evidence for a non-Gaussian cosmological model such as topological defects? Does it mean we cannot trust the data? Neither conclusion is justified (although both could be correct) because we do in fact expect a wide variation among these measurements due to their coverage of a very small portion of the sky. Just as it is difficult to measure the $C_{\ell}$ with only a few $a_{\ell m}$, it is challenging to use a small piece of the sky to measure multipoles whose spherical harmonics cover the sphere. It turns out that limited sky coverage leads to a sample variance for a particular multipole related to the cosmic variance for any value of $\ell$ by the simple formula

$$
\sigma_{s v}^{2} \simeq\left(\frac{4 \pi}{\Omega}\right) \sigma_{c v}^{2}
$$

where $\Omega$ is the solid angle observed (Scott et al., 1994). One caveat: in testing cosmological models, this cosmic and sample variance should be derived from the $C_{\ell}$ of the model, not the observed value of the data. The difference is typically small but will bias the analysis of forthcoming high-precision observations if cosmic and sample variance are not handled properly.

\subsection{Binning $C M B$ data}

Because there are so many measurements and the most important ones have the smallest error bars, it is preferable to plot the data in some way that 
avoids having the least precise measurements dominate the plot. Quantitative analyses should weight each datapoint by the inverse of its variance. Binning the data can be useful for display purposes but is dangerous for analysis, because a statistical analysis performed on the binned datapoints will give different results from one performed on the raw data. The distribution of the binned errors is non-Gaussian even if the original points had Gaussian errors. Binning might improve a quantitative analysis if the points at a particular angular scale showed a scatter larger than is consistent with their error bars, leading one to suspect that the errors have been underestimated. In this case, one could use the scatter to create a reasonable uncertainty on the binned average. For the current $\mathrm{CMB}$ data there is no clear indication of scatter inconsistent with the errors so this is unnecessary.

If one wishes to perform a model-dependent analysis of the data, the simplest reasonable approach is to compare the observations with the broad-band power estimates that should have been produced given a particular theory (the theory's $C_{\ell}$ are not constant so the window functions must be used for this). Combining full raw datasets is superior but computationally intensive (see Bond et al. 1998a). A first-order correction for the non-gaussianity of the likelihood function of the band-powers has been calculated by Bond et al. (1998b) and is available at http://www.cita.utoronto.ca/ knox/radical.html.

\section{Combining CMB and Large-Scale Structure Observations}

As CMB anisotropy is detected on smaller angular scales and large-scale structure surveys extend to larger regions, there is an increasing overlap in the spatial scale of inhomogeneities probed by these complementary techniques. This allows us to test the gravitational instability paradigm in general and then move on to finding cosmological models which can simultaneously explain the CMB and large-scale structure observations. Figure 5 shows this comparison for our compilation of CMB anisotropy observations (colored boxes) and of large-scale structure surveys (APM - Gaztañaga \& Baugh 1998, LCRS - Lin et al. 1996, Cfa2+SSRS2 - Da Costa et al. 1994, PSCZ - Tadros et al. 1999, APM clusters - Tadros et al. 1998) including measurements of the dark matter fluctuations from peculiar velocities (Kolatt \& Dekel, 1997) and the abundance of galaxy clusters (Viana \& Liddle, 1996; Bahcall et al., 1997). Plotting CMB anisotropy data as measurements of the matter power spectrum is a modeldependent procedure, and the galaxy surveys must be corrected for redshift distortions, non-linear evolution, and galaxy bias (see Gawiser \& Silk 1998 for detailed methodology.) Figure 5 is good evidence that the matter and radiation inhomogeneities had a common origin - the standard $\Lambda$ CDM model with a Harrison-Zel'dovich primordial power spectrum predicts both rather well. On the detail level, however, the model is a poor fit $\left(\chi^{2} /\right.$ d.o.f. $\left.=2.1\right)$, and no 


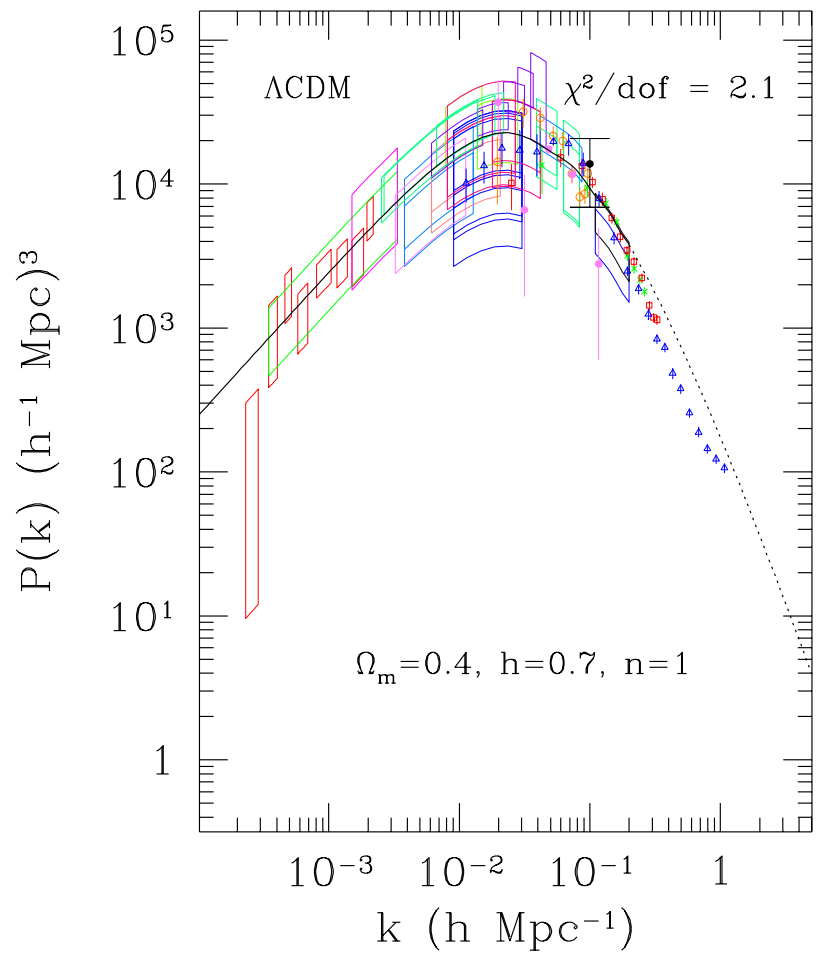

Fig. 5. Compilation of CMB anisotropy detections (boxes) and large-scale structure observations (points with error bars) compared to theoretical predictions of standard $\Lambda \mathrm{CDM}$ model. Height of boxes (and error bars) represents $1 \sigma$ uncertainties and width of boxes shows the full width at half maximum of each instrument's window function.

cosmological model which is consistent with the recent Type Ia supernovae results fits the data much better. Future observations will tell us if this is evidence of systematic problems in large-scale structure data or a fatal flaw of the $\Lambda$ CDM model.

\section{Conclusions}

The CMB is a mature subject. The spectral distortions are well understood, and the Sunyaev-Zeldovich effect provides a unique tool for studying galaxy clusters at high redshift. Global distortions will eventually be found, most 
likely first at very large $l$ due to the cumulative contributions from hot gas heated by radio galaxies, AGN, and galaxy groups and clusters. For gas at $\sim 10^{6}-10^{7} \mathrm{~K}$, appropriate to gas in galaxy potential wells, the thermal and kinematic contributions are likely to be comparable.

CMB anisotropies are a rapidly developing field, since the 1992 discovery with the COBE DMR of large angular scale temperature fluctuations. At the time of writing, the first acoustic peak is being mapped with unprecedented precision that will enable definitive estimates to be made of the curvature parameter. More information will come with all-sky surveys to higher resolution (MAP in 2000, PLANCK in 2007) that will enable most of the cosmological parameters to be derived to better than a few percent precision if the adiabatic CDM paradigm proves correct. Degeneracies remain in CMB parameter extraction, specifically between $\Omega_{0}, \Omega_{b}$ and $\Omega_{\Lambda}$, but these can be removed via large-scale structure observations, which effectively constrain $\Omega_{\Lambda}$ via weak lensing. The goal of studying reionization will be met by the interferometric surveys at very high resolution $\left(l \sim 10^{3}-10^{4}\right)$.

Polarization presents the ultimate challenge, because the foregrounds are poorly known. Experiments are underway to measure polarization at the 10 percent level, expected on degree scales in the most optimistic models. However one has to measure polarisation at the 1 percent level to definitively study the ionization history and early tensor mode generation in the universe, and this may only be possible with long duration balloon or space experiments.

CMB anisotropies are a powerful probe of the early universe. Not only can one hope to extract the cosmological parameters, but one should be able to measure the primordial power spectrum of density fluctuations laid down at the epoch of inflation, to within the uncertainties imposed by cosmic variance. In combination with new generations of deep wide field galaxy surveys, it should be possible to unambiguously measure the shape of the predicted peak in the power spectrum, and thereby establish unique constraints on the origin of the large-scale structure of the universe.

\section{References}

Aghanim, N., Desert, F. X., Puget, J. L., \& Gispert, R. 1996, Astron. Astrophys., 311,1

Albrecht, A. \& Steinhardt, P. J. 1982, Phys. Rev. Lett., 48, 1220

Bahcall, N. A., Fan, X., \& Cen, R. 1997, Astrophys. J., Lett., 485, L53

Baker, J. C. et al. 1999, preprint, astro-ph/9904415

Bennett, C. L., Banday, A. J., Gorski, K. M., Hinshaw, G., Jackson, P., Keegstra, P., Kogut, A., Smoot, G. F., Wilkinson, D. T., \& Wright, E. L. 1996, Astrophys. J., Lett., 464, L1 
Bond, J. R., Jaffe, A. H., \& Knox, L. 1998a, Phys. Rev. D, 57, 2117

-. 1998b, preprint, astro-ph/9808264

Boughn, S. P., Crittenden, R. G., \& Turok, N. G. 1998, New Astronomy, 3, 275

Bromley, B. C. \& Tegmark, M. 1999, Astrophys. J., Lett., 524, L79

Burigana, C. \& Popa, L. 1998, Astron. Astrophys., 334, 420

Cayon, L., Martinez-Gonzalez, E., \& Sanz, J. L. 1993, Astrophys. J., 403, 471

-. 1994, Astron. Astrophys., 284, 719

Church, S. E., Ganga, K. M., Ade, P. A. R., Holzapfel, W. L., Mauskopf, P. D., Wilbanks, T. M., \& Lange, A. E. 1997, Astrophys. J., 484, 523

Clapp, A. C., Devlin, M. J., Gundersen, J. O., Hagmann, C. A., Hristov, V. V., Lange, A. E., Lim, M., Lubin, P. M., Mauskopf, P. D., Meinhold, P. R., Richards, P. L., Smoot, G. F., Tanaka, S. T., Timbie, P. T., \& Wuensche, C. A. 1994, Astrophys. J., Lett., 433, L57

Coble, K. et al. 1999, preprint, astro-ph/9902195

Crittenden, R. G. \& Turok, N. 1996, Phys. Rev. Lett., 76, 575

Da Costa, L. N., Vogeley, M. S., Geller, M. J., Huchra, J. P., \& Park, C. 1994, Astrophys. J., Lett., 437, L1

De Oliveira-Costa, A., Devlin, M. J., Herbig, T., Miller, A. D., Netterfield, C. B., Page, L. A., \& Tegmark, M. 1998, Astrophys. J., Lett., 509, L77

De Oliveira-Costa, A., Kogut, A., Devlin, M. J., Netterfield, C. B., Page, L. A., \& Wollack, E. J. 1997, Astrophys. J., Lett., 482, L17

Dicker, S. R., Melhuish, S. J., Davies, R. D., Gutierrez, C. M., Rebolo, R., Harrison, D. L., Davis, R. J., Wilkinson, A., Hoyland, R. J., \& Watson, R. A. 1999, Mon. Not. R. Astron. Soc., 309, 750

Dodelson, S., Gates, E., \& Stebbins, A. 1996, Astrophys. J., 467, 10

Dodelson, S. \& Knox, L. 1999, preprint, astro-ph/9909454

Dwek, E., Arendt, R. G., Hauser, M. G., Fixsen, D., Kelsall, T., Leisawitz, D., Pei, Y. C., Wright, E. L., Mather, J. C., Moseley, S. H., Odegard, N., Shafer, R., Silverberg, R. F., \& Weiland, J. L. 1998, Astrophys. J., 508, 106

Femenia, B., Rebolo, R., Gutierrez, C. M., Limon, M., \& Piccirillo, L. 1998, Astrophys. J., 498, 117

Ferreira, P. G. \& Magueijo, J. 1997, Phys. Rev. D, 55, 3358

Ferreira, P. G., Magueijo, J., \& Gorski, K. M. 1998, Astrophys. J., Lett., 503, L1

Ferreira, P. G., Magueijo, J., \& Silk, J. 1997, Phys. Rev. D, 56, 4592

Fixsen, D. J., Dwek, E., Mather, J. C., Bennett, C. L., \& Shafer, R. A. 1998, Astrophys. J., 508, 123

Ganga, K., Page, L., Cheng, E., \& Meyer, S. 1994, Astrophys. J., Lett., 432, L15

Ganga, K., Ratra, B., Church, S. E., Sugiyama, N., Ade, P. A. R., Holzapfel, W. L., Mauskopf, P. D., \& Lange, A. E. 1997a, Astrophys. J., 484, 517

Ganga, K., Ratra, B., Gundersen, J. O., \& Sugiyama, N. 1997b, Astrophys. J., 484, 7

Ganga, K., Ratra, B., Lim, M. A., Sugiyama, N., \& Tanaka, S. T. 1998, Astrophys. J., Suppl. Ser., 114, 165

Gawiser, E., Finkbeiner, D., Jaffe, A., Baker, J. C., Balbi, A., Davis, M., Hanany, S., Holzapfel, W., Krumholz, M., Moustakas, L., Robinson, J., Scannapieco, E., Smoot, G. F., \& Silk, J. 1998, astro-ph/9812237

Gawiser, E., Jaffe, A., \& Silk, J. 1998, preprint, astro-ph/9811148 
Gawiser, E. \& Silk, J. 1998, Science, 280, 1405

Gawiser, E. \& Smoot, G. F. 1997, Astrophys. J., Lett., 480, L1

Gaztañaga, E. \& Baugh, C. M. 1998, Mon. Not. R. Astron. Soc., 294, 229

Gaztañaga, E., Fosalba, P., \& Elizalde, E. 1998, Mon. Not. R. Astron. Soc., 295, L35

Gruzinov, A. \& Hu, W. 1998, Astrophys. J., 508, 435

Gundersen, J. O. et al. 1995, Astrophys. J., Lett., 443, L57

Guth, A. 1981, Phys. Rev. D, 23, 347

Gutierrez, C. M. et al. 1999, preprint, astro-ph/9903196

Haimann, Z. \& Knox, L. 1999, in Microwave Foregrounds, ed. A. de Oliveira-Costa \& M. Tegmark (San Francisco: ASP), astro-ph/9902311

Halpern, M. \& Scott, D. 1999, in Microwave Foregrounds, ed. A. de Oliveira-Costa \& M. Tegmark (San Francisco: ASP), astro-ph/9904188

Holzapfel, W. L. et al. 1999, preprint, astro-ph/9912010

Hu, W. 1995, PhD thesis, U. C. Berkeley, astro-ph/9508126

Hu, W., Sugiyama, N., \& Silk, J. 1997, Nature, 386, 37

Hu, W. \& White, M. 1996, Phys. Rev. Lett., 77, 1687

-. 1997a, New Astronomy, 2, 323

-. 1997b, Astrophys. J., 479, 568

Jaffe, A., Gawiser, E., Finkbeiner, D., Baker, J. C., Balbi, A., Davis, M., Hanany, S., Holzapfel, W., Krumholz, M., Moustakas, L., Robinson, J., Scannapieco, E., Smoot, G. F., \& Silk, J. 1999, in Microwave Foregrounds, ed. A. de Oliveira Costa \& M. Tegmark (San Francisco: ASP), astro-ph/9903248

Kamionkowski, M. 1996, Phys. Rev. D, 54, 4169

Kamionkowski, M. \& Kinkhabwala, A. 1999, Phys. Rev. Lett., 82, 4172

Kamionkowski, M. \& Kosowsky, A. 1998, Phys. Rev. D, 57, 685

—. 1999, Annu. Rev. Nucl. Part. Sci., astro-ph/9904108

Kamionkowski, M., Kosowsky, A., \& Stebbins, A. 1997, Physical Review Letters, 78,2058

Kamionkowski, M., Spergel, D. N., \& Sugiyama, N. 1994, Astrophys. J., Lett., 426, L57

Knox, L. 1999, Phys. Rev. D, 60, 103516

Knox, L., Scoccimarro, R., \& Dodelson, S. 1998, Phys. Rev. Lett., 81, 2004

Kogut, A., Banday, A. J., Bennett, C. L., Gorski, K. M., Hinshaw, G., Jackson, P. D., Keegstra, P., Lineweaver, C., Smoot, G. F., Tenorio, L., \& Wright, E. L. 1996a, Astrophys. J., 470, 653

Kogut, A., Banday, A. J., Bennett, C. L., Gorski, K. M., Hinshaw, G., Smoot, G. F., \& Wright, E. L. 1996b, Astrophys. J., Lett., 464, L29

Kolatt, T. \& Dekel, A. 1997, Astrophys. J., 479, 592

Kolb, E. W. \& Turner, M. S. 1990, The Early Universe (Reading, MA: AddisonWesley Publishing Company)

Kosowsky, A. B. 1994, PhD thesis, Univ. of Chicago

Lee, A. T. et al. 1999, in proceedings of "3K Cosmology", ed. F. Melchiorri, astro$\mathrm{ph} / 9903249$

Leitch, E. M. et al. 1998, Astrophys. J., 518

Lin, H., Kirshner, R. P., Shectman, S. A., Landy, S. D., Oemler, A., Tucker, D. L., \& Schechter, P. L. 1996, Astrophys. J., 471, 617 
Linde, A. D. 1982, Phy. Lett., B108, 389

Martinez-Gonzalez, E., Sanz, J. L., \& Cayon, L. 1997, Astrophys. J., 484, 1

Masi, S., De Bernardis, P., De Petris, M., Gervasi, M., Boscaleri, A., Aquilini, E., Martinis, L., \& Scaramuzzi, F. 1996, Astrophys. J., Lett., 463, L47

Mason, B. S. et al. 1999, Astron. J., in press, preprint astro-ph/9903383

Mather, J. C. et al. 1994, Astrophys. J., 420, 439

Mauskopf, P. D. et al. 1999, preprint, astro-ph/9911444

Metcalf, R. B. \& Silk, J. 1997, Astrophys. J., 489, 1

—. 1998, Astrophys. J., Lett., 492, L1

Miller, A. D., Caldwell, R., Devlin, M. J., Dorwart, W. B., Herbig, T., Nolta, M. R., Page, L. A., Puchalla, J., Torbet, E., \& Tran, H. T. 1999, Astrophys. J., Lett., 524, L1

Netterfield, C. B., Devlin, M. J., Jarosik, N., Page, L., \& Wollack, E. J. 1997, Astrophys. J., 474, 47

Ostriker, J. P. \& Vishniac, E. T. 1986, Astrophys. J., Lett., 306, L51

Padmanabhan, T. 1993, Structure Formation in the Universe (Cambridge, UK: Cambridge University Press)

Pando, J., Valls-Gabaud, D., \& Fang, L. Z. 1998, Phys. Rev. Lett., 81, 4568

Partridge, R. B., Richards, E. A., Fomalont, E. B., Kellermann, K. I., \& Windhorst, R. A. 1997, Astrophys. J., 483, 38

Peebles, P. J. E. 1993, Principles of Physical Cosmology (Princeton, NJ: Princeton University Press)

Peebles, P. J. E. \& Juszkiewicz, R. 1998, Astrophys. J., 509, 483

Penzias, A. A. \& Wilson, R. W. 1965, Astrophys. J., 142, 419

Peterson, J. B. et al. 1999, preprint, astro-ph/9910503

Platt, S. R., Kovac, J., Dragovan, M., Peterson, J. B., \& Ruhl, J. E. 1997, Astrophys. J., Lett., 475, L1

Puget, J. L., Abergel, A., Bernard, J. P., Boulanger, F., Burton, W. B., Desert, F. X., \& Hartmann, D. 1996, Astron. Astrophys., 308, L5

Ratra, B., Ganga, K., Stompor, R., Sugiyama, N., de Bernardis, P., \& Gorski, K. M. 1999, Astrophys. J., 510, 11

Ratra, B., Ganga, K., Sugiyama, N., Tucker, G. S., Griffin, G. S., Nguyen, H. T., \& Peterson, J. B. 1998, Astrophys. J., 505, 8

Rees, M. J. \& Sciama, D. W. 1968, Nature, 217, 511

Refregier, A. 1999, in Microwave Foregrounds, ed. A. de Oliveira-Costa \& M. Tegmark (San Francisco: ASP), astro-ph/9904235

Refregier, A., Spergel, D. N., \& Herbig, T. 1998, preprint, astro-ph/9806349

Sachs, R. K. \& Wolfe, A. M. 1967, Astrophys. J., 147, 73

Schlegel, D. J., Finkbeiner, D. P., \& Davis, M. 1998, Astrophys. J., 500, 525

Scott, D., Srednicki, M., \& White, M. 1994, Astrophys. J., Lett., 421, L5

Scott, P. F. et al. 1996, Astrophys. J., Lett., 461, L1

Seljak, U. \& Zaldarriaga, M. 1997, Phys. Rev. Lett., 78, 2054

Seljak, U. \& Zaldarriaga, M. 1998, Phys. Rev. Lett., 82, 2636

Shu, F. H. 1991, The Physics of Astrophysics, Volume I: Radiation (Mill Valley, CA: University Science Books)

Silk, J. 1967, Nature, 215, 1155

Smoot, G. F., Bennett, C. L., Kogut, A., Wright, E. L., et al. 1992, Astrophys. J., 
Lett., 396, L1

Smoot, G. F., Gorenstein, M. V., \& Muller, R. A. 1977, Phys. Rev. Lett., 39, 898

Smoot, G. F. \& Scott, D. 1998, in Review of Particle Properties, astro-ph/9711069

Sokasian, A., Gawiser, E., \& Smoot, G. F. 1998, preprint, astro-ph/9811311

Staren, J. et al. 1999, preprint, astro-ph/9912212

Subrahmanyan, R., Ekers, R. D., Sinclair, M., \& Silk, J. 1993, Mon. Not. R. Astron. Soc., 263, 416

Suginohara, M., Suginohara, T., \& Spergel, D. N. 1998, Astrophys. J., 495, 511

Sunyaev, R. A. \& Zeldovich, Y. B. 1972, Astron. Astrophys., 20, 189

Tadros, H., Efstathiou, G., \& Dalton, G. 1998, Mon. Not. R. Astron. Soc., 296, 995

Tadros, H. et al. 1999, preprint, astro-ph/9901351

Tanaka, S. T. et al. 1996, Astrophys. J., Lett., 468, L81

Tegmark, M., Eisenstein, D. J., Hu, W., \& de Oliveira-Costa, A. 1999, preprint, astro-ph/9905257

Tegmark, M. \& Hamilton, A. 1997, preprint, astro-ph/9702019

Toffolatti, L., Argueso Gomez, F., De Zotti, G., Mazzei, P., Franceschini, A., Danese, L., \& Burigana, C. 1998, Mon. Not. R. Astron. Soc., 297, 117

Tolman, R. C. 1934, Relativity, Thermodynamics, and Cosmology (Oxford, UK: Clarendon Press)

Torbet, E., Devlin, M. J., Dorwart, W. B., Herbig, T., Miller, A. D., Nolta, M. R., Page, L., Puchalla, J., \& Tran, H. T. 1999, Astrophys. J., Lett., 521, L79

Tucker, G. S., Gush, H. P., Halpern, M., Shinkoda, I., \& Towlson, W. 1997, Astrophys. J., Lett., 475, L73

Viana, P. T. P. \& Liddle, A. R. 1996, Mon. Not. R. Astron. Soc., 281, 323

White, M. \& Bunn, E. 1995, Astrophys. J., Lett., 443, L53

White, M. \& Hu, W. 1997, Astron. Astrophys., 321, 8W

White, M., Scott, D., \& Silk, J. 1994, Ann. Rev. Astron. Astrophys., 32, 319

White, M. \& Srednicki, M. 1995, Astrophys. J., 443, 6

Wilson, G. W. et al. 1999, preprint, astro-ph/9902047

Yamada, M., Sugiyama, N., \& Silk, J. 1999, Astrophys. J., 522, 66

Zaldarriaga, M. \& Seljak, U. 1997, Phys. Rev. D, 55, 1830

Zaldarriaga, M. \& Seljak, U. 1998, preprint, astro-ph/9810257 
Table 1

Complete compilation of CMB anisotropy observations 1992-1999, with maximum likelihood $\Delta T$, upper and lower $1 \sigma$ uncertainties (not including calibration uncertainty), the weighted center of the window function, the $\ell$ values where the window function falls to $e^{-1 / 2}$ of its maximum value, the $1 \sigma$ calibration uncertainty, and references given below.

\begin{tabular}{|c|c|c|c|c|c|c|c|c|}
\hline Instrument & $\Delta T(\mu \mathrm{K})$ & $+1 \sigma(\mu \mathrm{K})$ & $-1 \sigma(\mu \mathrm{K})$ & $\ell_{e f f}$ & $\ell_{\min }$ & $\ell_{\max }$ & $1 \sigma$ cal. & ref. \\
\hline COBE1 & 8.5 & 16.0 & 8.5 & 2.1 & 2 & 2.5 & 0.7 & 1 \\
\hline COBE2 & 28.0 & 7.4 & 10.4 & 3.1 & 2.5 & 3.7 & 0.7 & 1 \\
\hline COBE3 & 34.0 & 5.9 & 7.2 & 4.1 & 3.4 & 4.8 & 0.7 & 1 \\
\hline COBE4 & 25.1 & 5.2 & 6.6 & 5.6 & 4.7 & 6.6 & 0.7 & 1 \\
\hline COBE5 & 29.4 & 3.6 & 4.1 & 8.0 & 6.8 & 9.3 & 0.7 & 1 \\
\hline COBE6 & 27.7 & 3.9 & 4.5 & 10.9 & 9.7 & 12.2 & 0.7 & 1 \\
\hline COBE7 & 26.1 & 4.4 & 5.3 & 14.3 & 12.8 & 15.7 & 0.7 & 1 \\
\hline COBE8 & 33.0 & 4.6 & 5.4 & 19.4 & 16.6 & 22.1 & 0.7 & 1 \\
\hline FIRS & 29.4 & 7.8 & 7.7 & 10 & 3 & 30 & $-^{a}$ & 2 \\
\hline TENERIFE & 30 & 15 & 11 & 20 & 13 & 31 & $\_^{a}$ & 3 \\
\hline IACB1 & 111.9 & 49.1 & 43.7 & 33 & 20 & 57 & 20 & 4 \\
\hline IACB2 & 57.3 & 16.4 & 16.4 & 53 & 38 & 75 & 20 & 4 \\
\hline SP91 & 30.2 & 8.9 & 5.5 & 57 & 31 & 106 & 15 & 5 \\
\hline SP94 & 36.3 & 13.6 & 6.1 & 57 & 31 & 106 & 15 & 5 \\
\hline BAM & 55.6 & 27.4 & 9.8 & 74 & 28 & 97 & 20 & 6 \\
\hline ARGO94 & 33 & 5 & 5 & 98 & 60 & 168 & 5 & 7 \\
\hline ARGO96 & 48 & 7 & 6 & 109 & 53 & 179 & 10 & 8 \\
\hline JBIAC & 43 & 13 & 12 & 109 & 90 & 128 & 6.6 & 9 \\
\hline QMAP(Ka1) & 47.0 & 6 & 7 & 80 & 60 & 101 & 12 & 10 \\
\hline QMAP(Ka2) & 59.0 & 6 & 7 & 126 & 99 & 153 & 12 & 10 \\
\hline QMAP(Q) & 52.0 & 5 & 5 & 111 & 79 & 143 & 12 & 10 \\
\hline MAX234 & 46 & 7 & 7 & 120 & 73 & 205 & 10 & 11 \\
\hline MAX5 & 43 & 8 & 4 & 135 & 81 & 227 & 10 & 12 \\
\hline MSAMI & 34.8 & 15 & 11 & 84 & 39 & 130 & 5 & 13 \\
\hline MSAMII & 49.3 & 10 & 8 & 201 & 131 & 283 & 5 & 13 \\
\hline MSAMIII & 47.0 & 7 & 6 & 407 & 284 & 453 & 5 & 13 \\
\hline
\end{tabular}




\begin{tabular}{|c|c|c|c|c|c|c|c|c|}
\hline Instrument & $\Delta T(\mu \mathrm{K})$ & $+1 \sigma(\mu \mathrm{K})$ & $-1 \sigma(\mu \mathrm{K})$ & $\ell_{e f f}$ & $\ell_{\min }$ & $\ell_{\max }$ & $1 \sigma$ cal. & ref. \\
\hline PYTHON123 & 60 & 9 & 5 & 87 & 49 & 105 & 20 & 14 \\
\hline PYTHON3S & 66 & 11 & 9 & 170 & 120 & 239 & 20 & 14 \\
\hline PYTHONV1 & 23 & 3 & 3 & 50 & 21 & 94 & $17^{b}$ & 15 \\
\hline PYTHONV2 & 26 & 4 & 4 & 74 & 35 & 130 & 17 & 15 \\
\hline PYTHONV3 & 31 & 5 & 4 & 108 & 67 & 157 & 17 & 15 \\
\hline PYTHONV4 & 28 & 8 & 9 & 140 & 99 & 185 & 17 & 15 \\
\hline PYTHONV5 & 54 & 10 & 11 & 172 & 132 & 215 & 17 & 15 \\
\hline PYTHONV6 & 96 & 15 & 15 & 203 & 164 & 244 & 17 & 15 \\
\hline PYTHONV7 & 91 & 32 & 38 & 233 & 195 & 273 & 17 & 15 \\
\hline PYTHONV8 & 0 & 91 & 0 & 264 & 227 & 303 & 17 & 15 \\
\hline $\mathrm{SK} 1^{c}$ & 50.5 & 8.4 & 5.3 & 87 & 58 & 126 & 11 & 16 \\
\hline SK2 & 71.1 & 7.4 & 6.3 & 166 & 123 & 196 & 11 & 16 \\
\hline SK3 & 87.6 & 10.5 & 8.4 & 237 & 196 & 266 & 11 & 16 \\
\hline SK4 & 88.6 & 12.6 & 10.5 & 286 & 248 & 310 & 11 & 16 \\
\hline SK5 & 71.1 & 20.0 & 29.4 & 349 & 308 & 393 & 11 & 16 \\
\hline TOCO971 & 40 & 10 & 9 & 63 & 45 & 81 & 10 & 17 \\
\hline TOCO972 & 45 & 7 & 6 & 86 & 64 & 102 & 10 & 17 \\
\hline TOCO973 & 70 & 6 & 6 & 114 & 90 & 134 & 10 & 17 \\
\hline TOCO974 & 89 & 7 & 7 & 158 & 135 & 180 & 10 & 17 \\
\hline TOCO975 & 85 & 8 & 8 & 199 & 170 & 237 & 10 & 17 \\
\hline TOCO981 & 55 & 18 & 17 & 128 & 102 & 161 & 8 & 18 \\
\hline TOCO982 & 82 & 11 & 11 & 152 & 126 & 190 & 8 & 18 \\
\hline TOCO983 & 83 & 7 & 8 & 226 & 189 & 282 & 8 & 18 \\
\hline TOCO984 & 70 & 10 & 11 & 306 & 262 & 365 & 8 & 18 \\
\hline TOCO985 & 24.5 & 26.5 & 24.5 & 409 & 367 & 474 & 8 & 18 \\
\hline VIPER1 & 61.6 & 31.1 & 21.3 & 108 & 30 & 229 & 8 & 19 \\
\hline VIPER2 & 77.6 & 26.8 & 19.1 & 173 & 72 & 287 & 8 & 19 \\
\hline VIPER3 & 66.0 & 24.4 & 17.2 & 237 & 126 & 336 & 8 & 19 \\
\hline VIPER4 & 80.4 & 18.0 & 14.2 & 263 & 150 & 448 & 8 & 19 \\
\hline VIPER5 & 30.6 & 13.6 & 13.2 & 422 & 291 & 604 & 8 & 19 \\
\hline VIPER6 & 65.8 & 25.7 & 24.9 & 589 & 448 & 796 & 8 & 19 \\
\hline
\end{tabular}




\begin{tabular}{|l|l|l|l|l|l|l|l|l|}
\hline Instrument & $\Delta T(\mu \mathrm{K})$ & $+1 \sigma(\mu \mathrm{K})$ & $-1 \sigma(\mu \mathrm{K})$ & $\ell_{\text {eff }}$ & $\ell_{\min }$ & $\ell_{\max }$ & $1 \sigma$ cal. & ref. \\
\hline BOOM971 & 29 & 13 & 11 & 58 & 25 & 75 & 8.1 & 20 \\
BOOM972 & 49 & 9 & 9 & 102 & 76 & 125 & 8.1 & 20 \\
BOOM973 & 67 & 10 & 9 & 153 & 126 & 175 & 8.1 & 20 \\
BOOM974 & 72 & 10 & 10 & 204 & 176 & 225 & 8.1 & 20 \\
BOOM975 & 61 & 11 & 12 & 255 & 226 & 275 & 8.1 & 20 \\
BOOM976 & 55 & 14 & 15 & 305 & 276 & 325 & 8.1 & 20 \\
BOOM977 & 32 & 13 & 22 & 403 & 326 & 475 & 8.1 & 20 \\
BOOM978 & 0 & 130 & 0 & 729 & 476 & 1125 & 8.1 & 20 \\
CAT96I & 51.9 & 13.7 & 13.7 & 410 & 330 & 500 & 10 & 21 \\
CAT96II & 49.1 & 19.1 & 13.7 & 590 & 500 & 680 & 10 & 21 \\
CAT99I & 57.3 & 10.9 & 13.7 & 422 & 330 & 500 & 10 & 22 \\
CAT99II & 0. & 54.6 & 0. & 615 & 500 & 680 & 10 & 22 \\
OVRO/RING & 56.0 & 7.7 & 6.5 & 589 & 361 & 756 & 4.3 & 23 \\
HACME & 0. & 38.5 & 0. & 38 & 18 & 63 & $-a$ & 29 \\
WD & 0. & 75.0 & 0. & 477 & 297 & 825 & 30 & 24 \\
SuZIE & 16 & 12 & 16 & 2340 & 1330 & 3070 & 8 & 25 \\
VLA & 0. & 27.3 & 0. & 3677 & 2090 & 5761 & $-a$ & 26 \\
ATCA & 0. & 37.2 & 0. & 4520 & 3500 & 5780 & $-a$ & 27 \\
BIMA & 8.7 & 4.6 & 8.7 & 5470 & 3900 & 7900 & $-a$ & 28 \\
\hline
\end{tabular}

REFERENCES: 1-Tegmark \& Hamilton (1997); Kogut et al. (1996a) 2-Ganga et al. (1994) 3-Gutierrez et al. (1999) 4-Femenia et al. (1998) 5-Ganga et al. (1997b); Gundersen et al. (1995) 6- Tucker et al. (1997) 7-Ratra et al. (1999) 8-Masi et al. (1996) 9-Dicker et al. (1999) 10-De Oliveira-Costa et al. (1998) 11-Clapp et al. (1994); Tanaka et al. (1996) 12-Ganga et al. (1998) 13-Wilson et al. (1999) 14Platt et al. (1997) 15-Coble et al. (1999) 16-Netterfield et al. (1997) 17-Torbet et al. (1999) 18-Miller et al. (1999) 19-Peterson et al. (1999) 20-Mauskopf et al. (1999) 21-Scott et al. (1996) 22-Baker et al. (1999) 23-Leitch et al. (1998) 24-Ratra et al. (1998) 25-Ganga et al. (1997a); Church et al. (1997) 26-Partridge et al. (1997) 27-Subrahmanyan et al. (1993) 28-Holzapfel et al. (1999) 29-Staren et al. (1999) ${ }^{a}$ Could not be determined from the literature.

${ }^{b}$ Results from combining the $+15 \%$ and $-12 \%$ calibration uncertainty with the $3 \mu \mathrm{K}$ beamwidth uncertainty. The non-calibration errors on the PYTHONV datapoints are highly correlated.

${ }^{c}$ The SK $\Delta T$ and error bars have been re-calibrated according to the $5 \%$ increase recommended by Mason et al. (1999) and the $2 \%$ decrease in $\Delta T$ due to foreground contamination found by De Oliveira-Costa et al. (1997). 